T. Matsuzawa

Nagoya Math. J.

Vol. 72 (1978), 103-126

\title{
CONSTRUCTION OF FUNDAMENTAL SOLUTIONS OF HYPOELLIPTIC EQUATIONS BY THE USE OF A PROBABILISTIC METHOD
}

\author{
T. MATSUZAWA
}

\section{§1. Introduction}

A. Friedman, [5] has constructed the fundamental solutions for a class of degenerate parabolic equations of the second order by making use of a probabilistic method and obtained the estimates for the fundamental solutions, especially near the degenerating manifolds (obstacles) of given operators, where a probabilistic method plays an important role.

Being suggested by this method, we shall discuss the case where the operators are hypoelliptic. Such a case is rather simple to be dealt with since there is no obstacle in the sense of A. Friedman. We shall consider the differential operator

$$
L \equiv \sum_{i, j=1}^{n} a_{i j}(x, s) \frac{\partial^{2}}{\partial x_{i} \partial x_{j}}+\sum_{j=1}^{n} b_{j}(x, s) \quad\left(a_{i j}=a_{j i}\right),
$$

where $a_{i j}(x, s)$ and $b_{j}(x, s)(i, j=1, \cdots, n)$ are real valued, infinitely differentiable functions defined in a neighborhood $\Omega$ of $\boldsymbol{R}^{n} \times[0, T]$, and assume :

I. The matrix $\left(a_{i j}(x, s)\right)$ is nonnegative definite for all $(x, s) \in \Omega$.

II. The coefficients $a_{i j}(x, s), \quad D_{x} a_{i j}(x, s), \quad D_{x}^{2} a_{i j}(x, s), \quad b_{j}(x, s)$ and $D_{x} b_{j}(x, s)(i, j=1, \cdots, n)$ are uniformly bounded in $\boldsymbol{R}^{n} \times[0, T]$.

III. The operators

$$
L+\frac{\partial}{\partial s}, \quad L^{*}-\frac{\partial}{\partial s}
$$

are hypoelliptic in $\Omega$, where $L^{*}$ is the formal adjoint operator of $L$.

Received October 4, 1977. 
Our first aim is to construct the fundamental solution of the equation

$$
L u+\frac{\partial u}{\partial s}=0
$$

under the assumptions I, II and III. For this purpose, we introduce the auxiliary equation

$$
L_{\varepsilon} u+\frac{\partial u}{\partial s}=0, \quad L_{s}=L+\varepsilon \Delta, \quad \Delta=\sum_{i=1}^{n} \frac{\partial^{2}}{\partial x_{i}}, \quad \varepsilon>0 .
$$

For the equation (1.2), being uniformly parabolic in $R^{n} \times[0, T]$, the classical fundamental solution $K_{\varepsilon}^{*}(x, s ; y, t)$ is constructed by the parametrix method [3]. In $\S 2$, we shall show that the fundamental solution $K^{*}(x, s ; y, t)$ of (1.1) is obtained by taking the limit of $K_{s}^{*}(x, s ; y, t)$ as $\varepsilon \rightarrow 0$. Let $c(x, t)$ be a bounded measurable function in $R^{n} \times[0, T]$. We shall construct the fundamental solution for the operator $L+c+\frac{\partial}{\partial s}$ in $\S 3$. In $\S 4$, as an application of the properties of $K^{*}$ obtained in $\S 2$, we shall prove the continuity of bounded generalized solutions of the equation

$$
\left[L+c+\frac{\partial}{\partial s}\right] u=f
$$

where $f$ is a given bounded measurable function, which is a main result of this paper. More restricted results have been obtained by the different method in [1]. In $\S 5$, we shall investigate the Cauchy problem (backward) for the equation (1.3). Finally in $\S 6$, we shall give some remarks on a first boundary value problem (Fichera problem).

\section{§2. Construction of fundamental solution}

As is mentioned in $\S 1$, the fundamental solution $K_{\varepsilon}^{*}(x, s ; y, t)$ is constructed by the parametrix method [3]. It satisfies the followings:

$$
\begin{gathered}
L_{\varepsilon} K_{\varepsilon}^{*}+\frac{\partial}{\partial s} K_{c}^{*}=0 \\
\text { in } R^{n}(0<s<t \leqq T) \text { for }(y, t) \text { fixed in } R^{n} \times[0, T] .
\end{gathered}
$$

We have $K_{c}^{*}(x, s ; y, t)>0$ for $0 \leqq s<t<T, K_{\varepsilon}^{*}(x, s ; y, t) \equiv 0$ for $0 \leqq t<s \leqq T$ and 


$$
\begin{cases}\int_{\boldsymbol{R}_{y}^{n}} K_{\varepsilon}^{*}(x, s ; y, t) d y=1 & \text { for }(x, s, t) \text { fixed in } \boldsymbol{R}^{n} \times[0, T]^{2}, s<t \\ \int_{\boldsymbol{R}_{x}^{n}} K_{\varepsilon}^{*}(x, s ; y, t) d x=1 & \text { for }(y, s, t) \text { fixed in } \boldsymbol{R}^{n} \times[0, T]^{2}, s<t .\end{cases}
$$

For any bounded continuous function $f(x)$ the function

$$
u(x, s)=\int_{R_{y}^{n}} K_{s}^{*}(x, s ; y, t) f(y) d y \quad 0<s<t \leqq T
$$

satisfies

$$
\left\{\begin{array}{l}
L_{\varepsilon} u+\frac{\partial u}{\partial s}=0 \quad \text { in } \boldsymbol{R}^{n} \times[0, t), \quad 0<t \leqq T, \\
u(x, s) \rightarrow f(x) \quad \text { as } s / t .
\end{array}\right.
$$

Now there exists uniquely, [2] a nonnegative definite matrix $\sigma(x, s)$ $=\left(\sigma_{i j}(x, s)\right)$ such that

$$
\frac{1}{2} \sigma(x, s)^{2}=\left(a_{i j}(x, s)\right), \quad(x, s) \in \boldsymbol{R}^{n} \times[0, T] .
$$

Each element of $\sigma(x, s)$ is Lipschitz continuous in any compact subset of $\boldsymbol{R}^{n} \times[0, T]$. Set $b=\left(b_{1}, \cdots, b_{n}\right)$ and consider the $n$-dimensional stochastic differential equations

$$
\left\{\begin{array}{l}
d \xi(t)=\sigma(\xi(t), t) d w(t)+b(\xi(t), t) d t \quad 0 \leqq s<t \leqq T, \\
\xi(s)=x, \quad x \in \boldsymbol{R}^{n},
\end{array}\right.
$$

where $w(t)=\left(w_{1}(t), \cdots, w_{n}(t)\right)$ is the $n$-dimensional Brownian motion. It is well known (c.f. [4]) that this system has a unique solution $\xi(t)$ $=\xi_{x, s}(t)$ for any initial conditions $\xi(s)=x \in \boldsymbol{R}^{n}$. The process $\xi(t)$ defines a diffusion process and gives the transition probability measure

$$
p(s, x, t, A)=P_{x, s}(\xi(t) \in A)=P\left(\xi_{x, s}(t) \in A\right),
$$

where $x \in \boldsymbol{R}^{n}, 0 \leqq s<t \leqq T$ and $A$ is a Borel set in $\boldsymbol{R}^{n}$. We shall denote by $B\left(\boldsymbol{R}^{n}\right)$ the $\sigma$-field of the Borel sets in $\boldsymbol{R}^{n}$ and introduce the notation

$$
S=\left\{(x, s) \in \boldsymbol{R}^{n} \times[0, T] ; \operatorname{det}\left(a_{i j}(x, s)\right)=0\right\} .
$$

THEOREM 2.1. Under the assumptions I, II and III, there exists a function $K^{*}(x, s ; y, t)$ infinitely differentiable in the complement of diagonal set of $\left(\boldsymbol{R}_{x}^{n} \times[0, T)\right] \times\left(\boldsymbol{R}_{y}^{n} \times[0, T]\right)$ with the following properties:

$$
K^{*}(x, s ; y, t)=\lim _{\varepsilon \rightarrow 0} K_{\varepsilon}^{*}(x, s ; y, t) \quad \text { in } \mathscr{D}^{\prime}\left(\boldsymbol{R}_{x}^{n} \times[0, T] \times \boldsymbol{R}_{y}^{n} \times[0, T]\right),
$$


from where we have $K^{*}(x, s ; y, t) \equiv 0$ for $0 \leqq t<s \leqq T$.

The convergence in (2.7) is uniform in any compact subset of

$\boldsymbol{R}_{x}^{n} \times \boldsymbol{R}_{y}^{n} \times(0 \leqq s<t \leqq T) \cap\{(x, s ; y, t) ;(x, s) \notin S,(y, t) \notin S\}$.

$$
\begin{aligned}
P_{x, s}(\xi(t) \in A)=\int_{A} K^{*}(x, s ; y, t) d y, & (x, s) \in \boldsymbol{R}^{n} \times[0, T], \\
& 0 \leqq s<t \leqq T, \quad A \in B\left(\boldsymbol{R}^{n}\right),
\end{aligned}
$$

in particular,

$$
\begin{gathered}
\int_{\boldsymbol{R}_{y}^{n}} K^{*}(x, s ; y, t) d y=1 \quad(x, s) \in \boldsymbol{R}^{n} \times[0, T], s<t \leqq T \\
\left(L_{x}+\frac{\partial}{\partial s}\right) K^{*}(x, s ; y, t)=-\delta(x-y, t-s), \\
\left(L_{y}^{*}-\frac{\partial}{\partial t}\right) K^{*}(x, s ; y, t)=-\delta(x-y, t-s),
\end{gathered}
$$

where $L^{*}$ is the formal adjoint of $L$.

If the coefficients $a_{i j}, b_{i}$ are independent of $t$, then $K^{*}(x, s ; y, t)$ $=K^{*}(x, 0 ; y, t-s) \equiv K(x, t-s, y) ; K(x, t, y)$ satisfies the equation

$$
\left(L-\frac{\partial}{\partial t}\right) K(x, t, y)=-\delta(x-y, t) \quad \text { in } \boldsymbol{R}_{x}^{n} \times \boldsymbol{R}_{y}^{n} \times[0, T] .
$$

THEOREM 2.2. Let $a_{i j}, b_{i}$ be independent of $t$ and in addition to the assumptions I, II and III we assume that $S_{0}=\left\{x \in \boldsymbol{R}^{n} ; \operatorname{det}\left(a_{i j}\right)=0\right\}$ is a compact set in $\boldsymbol{R}^{n}$. Then for any compact set $\boldsymbol{F}$ in $\boldsymbol{R}_{x}^{n} \times \boldsymbol{R}_{y}^{n} \backslash S_{0} \times S_{0}$ and for any positive numbers $T$ and $\varepsilon_{0}$, we have

$$
\begin{gathered}
0 \leqq K(x, t, y) \leqq C t^{-n / 2} \quad(x, y) \in F, 0<t<T, \\
0 \leqq K(x, t, y) \leqq C \exp \left(\frac{-c}{t}\right) \quad(x, y) \in F,|x-y| \geqq \varepsilon_{0}, 0<t<T,
\end{gathered}
$$

where $C$ and $c$ are positive constants. If $S_{0}$ consists of finite isolated points (2.14) and (2.15) hold for arbitrary compact set $\boldsymbol{F}$ in $\boldsymbol{R}_{x}^{n} \times \boldsymbol{R}_{y}^{n}$. Furthermore, for any compact set $E$ in $\boldsymbol{R}^{n}$, we can take $\rho$ sufficiently large so that we have

$$
0 \leqq K(x, t, y) \leqq C \exp \left[-\frac{c|x|^{2}}{t}\right] \quad y \in E, \quad|x|>\rho, \quad 0<t<T,
$$

where $C$ and $c$ are positive constants depending only on $E$ and $T$. 
THEOREM 2.3. Under the same assumptions as in Theorem 2.2, we have for sufficiently large $\rho$

$$
0 \leqq K(x, t, y) \leqq C \exp \left[-\frac{c|y|^{2}}{t}\right] \quad x \in E, \quad|y|>\rho, \quad 0<t<T,
$$

where $C$ and $c$ are positive constants depending only on $E$ and $T$.

We shall prove the above theorems in the following. First the proof of Theorem 2.1 will be given in several steps.

For each $\varepsilon>0$, consider the $n$-dimensional stochastic system

$$
\left\{\begin{array}{l}
d \xi^{\varepsilon}(t)=\sigma^{\varepsilon}\left(\xi^{\varepsilon}(t), t\right) d w(t)+b\left(\xi^{\mathrm{c}}(t), t\right) d t \quad 0 \leqq s<t \leqq T \\
\xi^{\mathrm{e}}(s)=x, \quad x \in \boldsymbol{R}^{n},
\end{array}\right.
$$

where $\sigma^{\varepsilon}$ is a matrix such that $\frac{1}{2} \sigma^{\varepsilon} \cdot \sigma^{\varepsilon}=\left(a_{i j}+\varepsilon \delta_{i j}\right)$.

The solution $\xi^{\varepsilon}(t)=\xi_{x, s}^{\varepsilon}(t)$ defines a diffusion process with the differential operator $L_{\varepsilon}+\frac{\partial}{\partial s}=L+\varepsilon \Delta+\frac{\partial}{\partial s}$. Since $L_{\varepsilon}+\frac{\partial}{\partial s}$ is uniformly parabolic (backward) in $\boldsymbol{R}^{n} \times[0, T]$ we have (c.f. [4])

$$
\begin{aligned}
P_{x, s}\left(\xi^{c}(t) \in A\right)= & \int_{A} K_{\varepsilon}^{*}(x, s ; y, t) d y \\
& x, y \in \boldsymbol{R}^{n}, 0 \leqq s<t \leqq T, A \in B\left(\boldsymbol{R}^{n}\right) .
\end{aligned}
$$

It can be shown that for any $N>0$ we have

$$
\sup _{\substack{0 \leq s<t \leq T \\|x| \leq N}} E_{x, s}\left|\xi^{s}(t)-\xi(t)\right| \rightarrow 0 \quad \text { as } \quad \varepsilon \rightarrow 0 \text {. (cf. [4], Vol. 1. Ch. 5) }
$$

As easily follows from the relation (2.19) we have for any $\varphi \in C_{0}^{\infty}\left(\boldsymbol{R}^{n}\right)$

$$
E_{x, s}\left[\varphi\left(\xi^{s}(t)\right)\right]=\int_{R_{y}^{n}} K_{\varepsilon}^{*}(x, s ; y, t) \varphi(y) d y \quad x \in \boldsymbol{R}^{n}, 0 \leqq s<t \leqq T .
$$

Now setting

$$
K_{s}^{*}(x, s ; y, t)=\left\{\begin{array}{cl}
\delta(x-y) & \text { for } t=s \\
0 & \text { for } t<s,
\end{array}\right.
$$

we have for any $\varphi(y, t) \in C_{0}^{\infty}\left(\boldsymbol{R}^{n} \times[0, T]\right)$

$$
\int_{s}^{T} E_{x, s}\left[\varphi\left(\xi^{\varepsilon}(t), t\right)\right] d t=\int_{0}^{T} \int_{R_{y}^{n}} K_{c}^{*}(x, s ; y, t) \varphi(y, t) d y d t
$$

Then we have from (2.20) 


$$
\begin{aligned}
& \left|\int_{s}^{T} E_{x, s}\left[\varphi\left(\xi^{\varepsilon}(t), t\right)\right] d t-\int_{s}^{T} E_{x, s}[\varphi(\xi(t), t)] d t\right| \\
& \quad \leqq \int_{s}^{T} E_{x, s}\left|\varphi\left(\xi^{c}(t), t\right)-\varphi(\xi(t), t)\right| d t \\
& \quad \leqq C(\varphi) \cdot T \cdot \sup _{s \leqq t \leqq T} E\left|\xi_{x, s}^{\epsilon}(t)-\xi_{x, s}(t)\right| \rightarrow 0 \quad \text { as } \varepsilon \rightarrow 0,
\end{aligned}
$$

where $C(\varphi)$ is a Lipschitz constant for $\varphi$. Thus we have for any $\varphi \in C_{0}^{\infty}\left(\boldsymbol{R}^{n} \times[0, T]\right)$

(2.23) $\int_{0}^{T} \int_{R_{y}^{n}} K_{\varepsilon}^{*}(x, s ; y, t) \varphi(y, t) d y d t \rightarrow \int_{s}^{T} E_{x, s}[\varphi(\xi(t), t)] d t \quad$ as $\varepsilon \rightarrow 0$,

$$
\sup _{(x, s) \in \boldsymbol{R}^{n} \times[0, T]}\left|\int_{0}^{T} \int_{\boldsymbol{R}_{y}^{n}} K_{\varepsilon}^{*}(x, s ; y, t) \varphi(y, t) d y d t\right| \leqq T \sup _{\boldsymbol{R}^{n} \times[0, T]}|\varphi| .
$$

On the other hand, it is well known that

$$
\left(L_{\varepsilon y}^{*}-\frac{\partial}{\partial t}\right) K_{\varepsilon}^{*}(x, s ; y, t)=0 \quad \text { for }(y, t) \neq(x, s),
$$

where $L_{\varepsilon}^{*}$ is the formal adjoint of $L_{\varepsilon}$. As a consequence of the above consideration, there exists a distribution $K^{*}$ such that

$$
\left(L_{y}^{*}-\frac{\partial}{\partial t}\right) K^{*}(x, s ; y, t)=-\delta(x-y, t-s) .
$$

Similarly we have

$$
\left(L_{x}+\frac{\partial}{\partial s}\right) K^{*}(x, s ; y, t)=-\delta(x-y, t-s) .
$$

By the assumption III and (2.25), we have for any fixed $(x, s) \in \boldsymbol{R}^{n} \times(0, T)$ $K^{*}(x, s ; y, t)$ is infinitely differentiable in $(y, t)$ in $\boldsymbol{R}^{n} \times(0, T) \backslash\{(x, s)\}$.

Now we can show that $K^{*}(x, s ; y, t)$ is infinitely differentiable in the complement of the diagonal set of $\left(\boldsymbol{R}_{x}^{n} \times(0, T)\right) \times\left(\boldsymbol{R}_{y}^{n} \times(0, T)\right)$ by the similar argument as in [1], $\S 6$. Take two disjoint bounded open sets $U$ and $V$ in $\boldsymbol{R}^{n} \times[0, T]$. Considering $(x, s) \in V$ as an parameter, $\left\{K^{*}(x, s ; y, t) ;(x, s) \in V\right\}$ is bounded in $\mathscr{D}^{\prime}(U)$ by (2.24). Furthermore by assumption III it is bounded in $C^{\infty}$-topology in $U$ (c.f. [1], Cor. 5.1). For each $T \in \mathscr{E}^{\prime}(U)$ set

$$
K^{*} T(x, s)=\left\langle T, K^{*}(x, s ; \cdot, \cdot)\right\rangle \quad(x, s) \in V .
$$

Take a sequence $\varphi_{n}(x, t) \in C_{0}^{\infty}(U)$ such that 


$$
\varphi_{n} \rightarrow T \quad \text { in } \mathscr{E}^{\prime}(U) \text { as } n \rightarrow \infty
$$

We have

$$
K^{*} \varphi_{n}(x, s)=\int_{0}^{T} \int_{R_{y}^{n}} K^{*}(x, s ; y, t) \varphi_{n}(y, t) d y d t \in C^{\infty}(V)
$$

and

$$
\left(L+\frac{\partial}{\partial s}\right) K^{*} \varphi_{n}=0 \quad \text { in } V:(2.26)
$$

On the other hand, we have $K^{*} \varphi_{n}(x, s) \rightarrow K^{*} T(x, s)$ for each $(x, s) \in V$ as $n \rightarrow \infty$, furthermore by the above consideration $K^{*} \varphi_{n}(x, s)$ converges boundedly to $K^{*} T(x, s)$ from where we have

$$
\left(L_{x}+\frac{\partial}{\partial s}\right) K^{*} T=0 \quad \text { in } V .
$$

Thus we have

$$
K^{*}: \mathscr{E}^{\prime}(U) \rightarrow \mathscr{E}(V)
$$

In such a case, by the results on distribution kernels given in [10], we have $K^{*} \in C^{\infty}(V \times U)$.

We note that from (2.23) we have for any $\varphi \in C_{0}^{\infty}\left(\boldsymbol{R}^{n}\right)$

$$
E_{x, s}[\varphi(\xi(t))]=\int_{R_{y}^{n}} K^{*}(x, s ; y, t) \varphi(y) d y
$$

from where we have $K^{*}(x, s ; y, t) \geqq 0$ and we can prove (2.9). In fact it is sufficient to prove (2.9) in the case where $A$ is an open ball. Take a sequence of functions $\varphi_{j}(x) \in C_{0}^{\infty}\left(\boldsymbol{R}^{n}\right)$ such that $\operatorname{supp} \varphi_{j} \subset A, j=1,2, \cdots$, and $\varphi_{j}(x) \nearrow 1$ for all $x \in A$ as $j \rightarrow \infty$. Then we have the relation (2.9) in such a case taking the limit as $j \rightarrow \infty$ for each side of (2.27) with $\varphi$ replaced by $\varphi_{j}$. The proof of Theorem 2.1 is completed except the assertion (2.8) which is proved by almost the same method given in [5]. We shall merely give the proof of (2.8) for a simple case in the proof of Theorem 2.2.

Now we are going to prove Theorem 2.2 and Theorem 2.3.

a) We shall first prove (2.14). Let $B$ be any bounded open set with $\bar{B} \cap S_{0}=\emptyset$. Then $K_{\varepsilon}(x, t, y)$ is a fundamental solution for $L_{\varepsilon}-$ $\frac{\partial}{\partial t}$ in the cylinder $Q=B \times(0, \infty)$. Since $L$ is non degenerate outside 
of $S_{0}$, we can easily see from the manner of the construction of $K_{c}$ (c.f. [3], p. 24) there is a constant $C$ independent of $\varepsilon(0<\varepsilon \leqq 1)$ such that

$$
0<K_{\varepsilon}(x, t, y) \leqq C t^{-n / 2} \quad x, y \in B, 0<t \leqq T .
$$

Thus for any sequence $\left\{\varepsilon_{m}\right\}, \varepsilon_{m} \rightarrow 0$ as $m \rightarrow \infty$, and for any compact set $W$ in $\left(\boldsymbol{R}_{x}^{n} \backslash S_{0}\right) \times(0, \infty) \times\left(\boldsymbol{R}_{y}^{n} \backslash S_{0}\right), K_{\varepsilon_{m}}$ is uniformly bounded in $W$. On the other hand we have

$$
\left(L_{\varepsilon_{m}, x}+L_{s_{m}, y}^{*}-2 \frac{\partial}{\partial t}\right) K_{\varepsilon_{m}}(x, t, y)=0 \quad \text { in } W
$$

and $L_{\varepsilon, x}+L_{\varepsilon, y}-2 \frac{\partial}{\partial t}(\varepsilon>0)$ is uniformly parabolic in $W$. Then by the Schauder type interior estimates (c.f. [3]) applied to $K_{\varepsilon_{m}}$ as a function of $(x, t, y)$ we conclude, by diagonalization, that there is a subsequence $\left\{\varepsilon_{m}^{\prime}\right\}$ of $\left\{\varepsilon_{m}\right\}$ such that

$$
K(x, t, y)=\lim _{m \rightarrow \infty} K_{\varepsilon_{m}^{\prime}}(x, t, y) \quad x \in \boldsymbol{R}^{n} \backslash S_{0}, y \in \boldsymbol{R}^{n} \backslash S_{0}, 0<t<\infty,
$$

where the convergence is uniform in any compact set in $\left(\boldsymbol{R}_{x}^{n} \backslash S_{0}\right) \times(0, \infty)$ $\times\left(\boldsymbol{R}_{y}^{n} \backslash S_{0}\right)$. This proves the assertion (2.8) in such a case.

Next let $Q$ be any compact set in $R^{N} \backslash S_{0}$ then by (2.28) and (2.29) we have

$$
0 \leqq K(x, t, y) \leqq C t^{-n / 2} \quad x \in Q, y \in Q, 0<t<T,
$$

where the positive constant $C$ depends only on $Q$ and $T$. Hence the estimate (2.14) is obvious if the estimate (2.15) is proved.

b) Now we shall prove (2.15). For any compact set $E$ with $E \cap S_{0}$ $=\emptyset$, we can see by the way of construction of $K_{\varepsilon}$ (c.f. [3], Ch. 1) that

$$
0<K_{\varepsilon}(x, t, y) \leqq C \exp \left(-\frac{c}{t}\right) \quad x \in E, y \in E,|x-y| \geqq \varepsilon_{0}, 0<t<T
$$

where $C$ and $c$ are positive constants depending only on $E, \varepsilon_{0}$ and $T$. Then by (2.29) we have the estimate (2.31) with $K_{\varepsilon}$ replaced by $K$. Let $M$ and $E$ be arbitrary bounded open sets in $R^{n}$ such that $S_{0} \subset M$, $\bar{M} \cap \bar{E}=\emptyset$. Suppose that the boundaries of $M$ and $E$ are smooth. Then by the argument given above we have

$$
0 \leqq K(x, t, y) \leqq C \exp \left(-\frac{c}{t}\right) \quad x \in \partial M, y \in E, 0<t \leqq T .
$$


For any fixed $y$ in $E$ consider the function

$$
v(x, t) \equiv K(x, t, y) \quad \text { in }(x, t) \in M \times(0, T) .
$$

Then we have by (2.32) and (2.7)

$$
\left\{\begin{array}{l}
0 \leqq v(x, t) \leqq C \exp \left(-\frac{c}{t}\right) \quad(x, t) \in \partial M \times(0, T), \\
v(x, 0)=0 \quad x \in M, \\
\left(L-\frac{\partial}{\partial t}\right) v(x, t)=0 \quad \text { in } M \times(0, T) .
\end{array}\right.
$$

Hence by the maximum principle it follows that

$$
0 \leqq v(x, t) \leqq C \exp \left(-\frac{c}{t}\right) \quad(x, t) \in M \times(0, T),
$$

i.e.,

$$
0 \leqq K(x, t, y) \leqq C \exp \left(-\frac{c}{t}\right) \quad x \in M, 0<t \leqq T, y \in E
$$

Next take any bounded open sets $M$ and $E$ such that $S_{0} \subset E$ and $\bar{M} \cap \bar{E}$ $=\emptyset$ and consider the function

$$
v(t, y) \equiv K(x, t, y) \quad \text { in }(t, y) \in(0, T) \times E
$$

for any fixed $x \in M$. Then by the similar way as above we have the estimate of type (2.33). Combining these investigations we have (2.15). It is clear, from the method given above, that (2.14) and (2.15) hold for arbitrary compact set $F$ if $S_{0}$ consists of finite isolated points.

c) We shall prove the estimate (2.16) by the similar way as in [5], $\S 4$. Let $B_{m}=\left\{x \in \boldsymbol{R}^{n} ;|x|<m\right\}, m=1,2, \cdots$. Denote by $G_{m, \varepsilon}(x, t, y)$ the Green function for the operator

$$
L_{\varepsilon}-\frac{\partial}{\partial t}=\sum_{i, j=1}^{n} a_{i j}(x) \frac{\partial^{2}}{\partial x_{i} \partial x_{j}}+\sum_{j=1}^{n} b_{j}(x) \frac{\partial}{\partial x_{j}}+\varepsilon \Delta-\frac{\partial}{\partial t}
$$

in the cylinder $Q_{m}=B_{m} \times(0, \infty)$. Therefore $G_{m, s}(x, t, y) \in C^{\infty}\left(\bar{B}_{m} \times(0, \infty)\right.$ $\left.\times B_{m}\right)$ and as a function of $(x, t)$ we have

$$
\begin{gathered}
L_{\varepsilon} G_{m, \varepsilon}-\frac{\partial}{\partial t} G_{m, \varepsilon}=0 \quad \text { in }(x, t) \in Q_{m}\left(y \text { fixed in } B_{m}\right), \\
G_{m, \varepsilon} \rightarrow 0 \quad \text { as } t \rightarrow 0 \text { if } x \neq y, x \in B_{m}, \\
G_{m, \varepsilon}=0 \quad \text { if } t \rightarrow 0, x \in \partial B_{m} .
\end{gathered}
$$


Furthermore, for any continuous function $f(y)$ with support in $B_{m}$, the function

$$
u(x, t)=\int_{B_{m}} G_{m, e}(x, t, y) f(y) d y
$$

satisfies :

$$
\left\{\begin{array}{l}
L_{\varepsilon} u(x, t)-\frac{\partial}{\partial t} u=0 \quad \text { in } Q_{m}, \\
u(x, t) \rightarrow f(x) \quad \text { as } t \rightarrow 0, x \in B_{m}, \\
u(x, t)=0 \quad \text { for } t \rightarrow 0, x \in \partial B_{m} .
\end{array}\right.
$$

It is well known (c.f. [3], p. 82) that such a function $G_{m, s}$ is constructed by using $K_{\varepsilon} \equiv K_{\varepsilon}^{*}(x, 0 ; t, y)$ and is uniquely determined by the above properties. We have

$$
\begin{gathered}
0 \leqq G_{m, \varepsilon} \leqq G_{m+1, \varepsilon} \leqq K_{\varepsilon}(x, t, y) \quad \text { if }(x, t) \in Q_{m}, y \in B_{m} \\
K_{\varepsilon}(x, t, y)=\lim _{m \rightarrow \infty} G_{m, \varepsilon}(x, t, y) \quad x, y \in R^{n}, t>0
\end{gathered}
$$

The inequalities in (2.35) are proved by the maximum principle. In fact for any continuous and nonnegative function $f_{k}(y)$ with support in $B_{m}$, we have

$$
\begin{aligned}
0 & \leqq \int_{B_{m}} G_{m, \varepsilon}(x, t, y) f_{k}(y) d y \leqq \int_{B_{m+1}} G_{m+1, \varepsilon}(x, t, y) f_{k}(y) d y \\
& \leqq \int_{\boldsymbol{R}_{y}^{n}} K_{\varepsilon}(x, t, y) f_{k}(y) d y
\end{aligned}
$$

by the maximum principle. Taking a sequence $f_{k}$ converging to the Dirac measure at $y \in B_{m}$, the inequalities in (2.35) follow. Now we can use the Schauder type estimate as in a) to conclude that the following limit exists :

$$
G_{\epsilon}(x, t, y)=\lim _{m \rightarrow \infty} G_{m, \varepsilon}(x, t, y) \quad x, y \in \boldsymbol{R}^{n}, t>0 .
$$

The convergence in (2.17) is uniform in any compact set of $\boldsymbol{R}_{x}^{n} \times(0, \infty)$ $\times \boldsymbol{R}_{y}^{n}$. Setting

$$
G_{\varepsilon}(x, t, y)=\left\{\begin{array}{cc}
\delta(x-y) & \text { for } t=0 \\
0 & \text { for } t \rightarrow 0
\end{array}\right.
$$

we can easily see that 


$$
\left(L_{\epsilon, x}-\frac{\partial}{\partial t}\right) G_{\imath}(x, t, y)=\delta(x-y, t) .
$$

Recalling that $K_{\varepsilon}$ has the same property as $G_{\varepsilon}$, we have for any fixed $y \in \boldsymbol{R}^{n}$ that

$$
\left\{\begin{array}{l}
\left(L_{\varepsilon, x}-\frac{\partial}{\partial t}\right)\left(G_{\varepsilon}-K_{\varepsilon}\right)=0 \quad t>0, \\
\left(G_{\varepsilon}-K_{\varepsilon}\right)_{t=0}=0 \\
\left|G_{\varepsilon}-K_{\varepsilon}\right| \leqq 2 K_{\varepsilon} .
\end{array}\right.
$$

Hence we have $G_{\varepsilon}-K_{\varepsilon} \equiv 0$ by the well known uniqueness theorem for the Cauchy problem for uniformly parabolic equations. This proves (2.36). After these preparations we can prove the estimate (2.16). Consider first the case where $E \cap S=\emptyset$. For any positive integers $\rho$ and $m, m>\rho$, let

$$
N_{m, \rho}=\left\{x \in \boldsymbol{R}^{n} ; \rho<|x|<m\right\}, \quad \Delta_{\rho}=\{x ;|x|=\rho\}, \quad \Delta_{m}=\{x ;|x|=m\} .
$$

We shall compare the function $v(x, t)=G_{m, c}(x, t, y)$ (y fixed in $E$ ) with a function

$$
w(x, t)=C \exp \left(-\frac{\gamma}{t}|x|^{2}\right)
$$

in the cylinder $N_{m, \rho} \times(0, T)$. We have

$$
\begin{aligned}
t^{2}\left(L_{\varepsilon}-\frac{\partial}{\partial t}\right) w(x, t)= & \left\{4 \gamma^{2} \sum_{i, j=1}^{n}\left(a_{i j}+\varepsilon \delta_{i j}\right) x_{i} x_{j}-2 \gamma t\left[\sum_{i=1}^{n}\left(a_{i i}+\varepsilon\right)\right.\right. \\
& \left.+\sum_{i=1}^{n} b_{i} x_{i}\right]-\gamma|x|^{2} C \exp \left(-\frac{\gamma}{t}|x|^{2}\right) .
\end{aligned}
$$

We have $L_{\varepsilon} w-w_{t}<0$ for $(x, t) \in N_{m, \rho} \times(0, T)$ if we take $\gamma$ sufficiently small and $\rho$ sufficiently large which can be taken independent of $\gamma$ and $\varepsilon$ by the assumption II. With $\rho$ now fixed, we further decrease $\gamma$ (if necessary) so that

$$
v(x, t) \leqq w(x, t) \quad \text { for } x \in \Delta_{\rho}, 0<t<T
$$

for some positive constant $C$ in (2.38), which is possible by virtue of (2.31). Notice that $v(x, 0)=0$ by (2.31) and applying the maximum principle, we get

$$
G_{m, e}(x, t, y)=v(x, t) \leqq w(x, t) \quad \text { for }(x, t) \in N_{m, \rho} \times(0, T) .
$$


From this the assertion (2.16) for such a case follows by taking first $m \rightarrow \infty$ and then $\varepsilon \rightarrow 0$.

Now let $E$ be any compact set in $\boldsymbol{R}^{n}$. Let $\Sigma$ be a sphere containing both $E$ and $S_{0}$ in its interior $\Delta$. From what we have just proved we know that for $x \in N_{m, \rho}$

$$
G_{m, \epsilon}(x, t, y) \leqq w(x, t) \quad(y, t) \in \Sigma \times(0, T) .
$$

Considering as a function of $(y, t), w(x, t)$ satisfies :

$$
\left(L_{\varepsilon, y}^{*}-\frac{\partial}{\partial t}\right) w \equiv\left(\tilde{c}(y)-\frac{\gamma}{t^{2}}|x|^{2}\right) w(x, t)<0
$$

if $\rho$ is sufficiently large and $y \in \Delta, 0<t<T$. By using the maximum principle we have (2.39) also for $y \in \Delta, 0<t<T$. Taking $m \rightarrow \infty$ and then $\varepsilon \rightarrow 0$, (2.16) follows.

d) As easily seen, we can apply the proof of Theorem 2.2 to the adjoint $L^{*}$ of $L$ and obtain Theorem 2.3.

\section{$\S 3$. Fundamental solution for the operator $L+c(x, s)+\frac{\partial}{\partial s}$}

1. Let $L$ satisfy the assumptions I, II and III given in $\S 1$. Let $c(x, t)$ be a bounded measurable function in $\boldsymbol{R}_{x}^{n} \times[0, T]$ and we shall construct a solution of the equation

$$
\left(L+c(x, s)+\frac{\partial}{\partial s}\right) \Gamma^{*}(x, s ; y, t)=-\delta(x-y, t-s) .
$$

First we suppose $c(x, s) \geqq 0$ and we try to find $\Gamma^{*}$ in the form

$$
\begin{array}{r}
\Gamma^{*}(x, s ; y, t)=K^{*}(x, s ; y, t)+\int_{s}^{t} \int_{R_{\xi}^{n}} K^{*}(x, s ; \xi, \sigma) \Phi^{*}(\xi, \sigma ; y, t) d \xi d \sigma \\
0 \leqq s<t \leqq T,
\end{array}
$$

and we define $\Gamma^{*}(x, s ; y, t) \equiv 00 \leqq t<s \leqq T$.

The integral in the right hand side of (3.2) has a meaning by the Chapman-Kolmogorov's equation if we have

$$
0 \leqq \Phi^{*}(x, s ; y, t) \leqq C K^{*}(x, s ; y, t) \quad 0 \leqq s<t \leqq T,
$$

where $C$ is a positive constant. Suppose there exists such a solution $\Gamma^{*}$, then if we operate $L+c(x, s)+\frac{\partial}{\partial s}$ for both sides of (3.2) in the 
distribution sense we have

$$
\begin{aligned}
& -\delta(x-y ; t-s)=-\delta(x-y ; t-s)+c(x, s) K^{*}(x, s ; y, t) \\
& \quad-\Phi^{*}(x, s ; y, t)+c(x, s) \int_{s}^{t} \int_{R_{\xi}^{n}} K^{*}(x, s ; \xi, \sigma) \Phi^{*}(\xi, \sigma ; y, t) d \xi d \sigma .
\end{aligned}
$$

Hence the problem is reduced to solve the integral equation

$$
\begin{aligned}
& \Phi^{*}(x, s ; y, t)=c(x, s)\left[K^{*}(x, s ; y, t)\right. \\
& \left.\quad+\int_{s}^{t} \int_{R_{\xi}^{n}} K^{*}(x, s ; \xi, \sigma) \Phi^{*}(\xi, \sigma ; y, t) d \xi d \sigma\right],
\end{aligned}
$$

which will be solved by recursive approximation. Define

$$
\Phi_{0}^{*}(x, s ; y, t)=c(x, s) K^{*}(x, s ; y, t)
$$

and

$$
\Phi_{k}^{*}(x, s ; y, t)=c(x, s) \int_{s}^{t} \int_{R_{\xi}^{n}} K(x, s ; \xi, \sigma) \Phi_{k-1}^{*}(\xi, \sigma ; y, t) d \xi d \sigma \quad k=1,2, \cdots
$$

Then we have

$$
0 \leqq \Phi_{k}^{*}(x, s ; y, t) \leqq M^{k+1} \frac{(t-s)^{k}}{k !} K^{*}(x, s ; y, t),
$$

where $M=\sup c(x, s)$ in $\boldsymbol{R}^{n} \times[0, T]$. Hence the solution $\Phi^{*}$ of (3.4) is obtained by

$$
\Phi^{*}(x, s ; y, t)=\sum_{k=0}^{\infty} \Phi_{k}^{*}(x, s ; y, t)
$$

and we have

$$
0 \leqq \Phi^{*}(x, s ; y, t) \leqq M e^{(t-s) M} K^{*}(x, s ; y, t), \quad 0 \leqq s<t \leqq T .
$$

Thus we have obtained the fundamental solution $\Gamma^{*}$ of the operator $L+c(x, s)+\frac{\partial}{\partial s}$ and it holds that

$$
0 \leqq \Gamma^{*}(x, s ; y, t) \leqq e^{(t-s) M} K^{*}(x, s ; y, t), \quad 0 \leqq s<t \leqq T .
$$

Now let $c(x, t)$ be an arbitrary bounded measurable function in $\boldsymbol{R}^{n} \times[0, T]$ then we can construct as above the fundamental solution $\Gamma_{\mu}^{*}$ for the operator $L+c(x, s)+\mu+\frac{\partial}{\partial s}$ with $\mu=-\inf c(x, s)$ in $R^{n} \times$ $[0, T]$. By (3.8) we have 


$$
0 \leqq \Gamma_{\mu}^{*}(x, s ; y, t) \leqq e^{(M+\mu)(t-s)} K^{*}(x, s ; y, t), \quad M=\sup _{\boldsymbol{R}^{n} \times[0, T]} c(x, s)
$$

The fundamental solution of the operator $L+c+\frac{\partial}{\partial s}$ is given by $\Gamma^{*}$ $=e^{-\mu(t-s)} \Gamma_{\mu}^{*}$ and we have the estimate

$$
0 \leqq \Gamma^{*}(x, s ; y, t) \leqq e^{M(t-s)} K^{*}(x, s ; y, t) \quad 0 \leqq s<t \leqq T .
$$

2. Let $L=\sum_{i, j=1}^{n} a_{i j}(x) \frac{\partial^{2}}{\partial x_{i} \partial x_{j}}+\sum_{i=1}^{n} b_{i}(x)$ be independent of $t$ and satisfy the assumption in Theorem 2.2 and let $c(x)$ be a bounded measurable function in $\boldsymbol{R}^{n}$. We shall construct a fundamental solution for the operator $L+c(x)-\lambda$ in $\boldsymbol{R}^{n}$ where $\lambda$ is a real number such that $\lambda>$ $\sup _{x \in R^{n}} c(x)$. We note that the fundamental solutions $K(x, t, y) \equiv K^{*}(x, 0 ; y, t)$ and $\Gamma(x, t, y)=\Gamma^{*}(x, 0 ; y, t)$ for the operators $L-\frac{\partial}{\partial t}$ and $L+c-\frac{\partial}{\partial t}$ have been constructed in the paragraph 1. For any positive numbers $t$ and $s_{0}$ we have

$$
K\left(x, t+s_{0}, y\right)=\int_{R_{\xi}^{n}} K(x, t, \xi) K\left(\xi, s_{0}, y\right) d \xi .
$$

Let $\mathrm{E}$ be any compact set in $\boldsymbol{R}^{n}$ then by applying (2.10) and (2.16) in the right hand side of (3.10), we have

$$
0 \leqq K\left(x, t+s_{0}, y\right) \leqq M_{0} \quad(x, t) \in \boldsymbol{R}^{n} \times(0, \infty), y \in E,
$$

where $M_{0}$ is a positive constant depending only on $E$ and $s_{0}$. Therefore the function

$$
G(x, y)=\int_{0}^{\infty} e^{-\lambda s} \Gamma(x, s, y) d s
$$

is well defined for $x=y$ by virtue of the fact that $K(x, t, y) \in C^{\infty}\left(\boldsymbol{R}_{x}^{n} \times\right.$ $(-\infty, \infty) \times \boldsymbol{R}_{y}^{n} \backslash\{x=y, t=0\}: \quad$ Theorem 2.2 and $0 \leqq \Gamma(x, t, y) \leqq$ $e^{(M-\lambda) t} K(x, t, y) t>0:$ (3.9). As is easily seen, it holds that

$$
(L+c(x)-\lambda) G(x, y)=-\delta(x-y) .
$$

Hence $-G(x, y)$ is a fundamental solution for the operator $L+c(x)-\lambda$ in $\boldsymbol{R}^{N}$.

Finally we note that the singularity of $G(x, y)$ is uniformly integrable, that is, 


$$
\int_{R_{y}^{n}} G(x, y) d y=\int_{R_{y}^{n}} \int_{0}^{\infty} e^{(M-\lambda) t} K(x, t, y) d y d t \leqq \frac{1}{\lambda-M} \quad x \in R^{n}
$$

\section{§4. Continuity of bounded generalised solutions}

Let $\Omega$ be a bounded open set in $R^{n}$. We shall consider the continuity property of solutions of the equation

$$
\left(L+c(x, s)+\frac{\partial}{\partial s}\right) u(x, s)=f(x, s) \quad \text { in } \Omega \times(0, T),
$$

where the operator $L=\sum_{i, j=1}^{n} a_{i i}(x, s) \frac{\partial^{2}}{\partial x_{i} \partial x_{j}}+\sum_{j=1}^{n} b_{j}(x, s) \frac{\partial}{\partial x_{j}}$ satisfies the conditions I, II and III given in $\S 1$ in $\Omega \times[0, T]$ and $c(x, s)$ is a bounded measurable function in $\Omega \times[0, T]$.

THEOREM 4.1. Let $u$ and $f$ be bounded measurable functions in $\Omega \times(0, T)$ which satisfy the equation (4.1) in $\Omega \times(0, T)$ in the sence of distributions. Then $u$ is a continuous function in $\Omega \times(0, T)$.

Proof. It is sufficient to prove the theorem in the case $c \equiv 0$ since we have $\left(L+\frac{\partial}{\partial s}\right) u=-c u+f$ and the right hand side is bounded measurable in $\Omega \times(0, T)$. For simplicity we assume that $\Omega$ is an open ball $B^{N}=\left\{x \in \boldsymbol{R}^{n} ;|x|<N\right\}$.

We shall first treat the case where supp. $f \subset B^{N} \times(0, T)$. We note that the coefficients $a_{i j}, b_{j}(i, j=1, \cdots, n)$ can be extended to be infinitely differentiable bounded functions in $\in \boldsymbol{R}^{n} \times[0, T]$ so that $L$ is elliptic in $\left(\boldsymbol{R}^{n} \backslash \bar{B}^{N}\right) \times[0, T]$. Then the conditions I and II are satisfied for extended $L$, and by the same proof as in Theorem 2.1 there exists a distribution kernel $K^{*}(x, s ; y, t)$ such that

$K^{*}$ is infinitely differentiable in the complement of the diagonal set of $\left(B_{x}^{N} \times[0, T]_{s}\right) \times\left(B_{y}^{N} \times[0, T]_{t}\right)$,

$$
\begin{aligned}
& \left(L+\frac{\partial}{\partial s}\right) K^{*}(x, s ; y, t)=-\delta(x-y, t-s) \quad \text { in } B^{N} \times[0, T] \\
& P_{x, s}(\xi(t) \in A)=\int_{A} K^{*}(x, s ; y, t) d y \quad 0 \leqq s<t \leqq T, \\
& \quad \text { for any }(x, s) \in B^{N} \times[0, T] \text { and } A \in B\left(B^{N}\right), \bar{A} \subset B^{N}
\end{aligned}
$$


Consider now the function

$$
u(x, s)=-\int_{0}^{T} \int_{B_{y}^{N}} K^{*}(x, s ; y, t) f(y, t) d y d t \quad(x, s) \in B^{N} \times[0, T] .
$$

Clearly we have by (4.4)

$$
\|u\|_{L^{\infty}\left(B^{N} \times[0, T]\right)} \leqq T\|f\|_{L^{\infty}\left(B^{N} \times\left[0, T^{\top}\right]\right)} .
$$

We shall show that

$$
\left(L+\frac{\partial}{\partial s}\right) u(x, s)=f(x, s) \quad \text { in } B^{N} \times[0, T]
$$

in the sense of distributions. In fact, take a sequence of functions $\varphi_{j}(x, s) \in C_{0}^{\infty}\left(B_{N} \times[0, T]\right)$ such that

$$
\begin{gathered}
\lim _{j \rightarrow \infty} \varphi_{j}(x, s)=f(x, s) \quad(x, s) \in B^{N} \times[0, T], \\
\left\|\varphi_{j}\right\|_{L^{\infty}} \leqq\|f\|_{L^{\infty}}, \quad j=1,2, \cdots .
\end{gathered}
$$

Then we have by (4.3)

$$
\begin{gathered}
\left(L+\frac{\partial}{\partial s}\right) u_{j}(x, s)=\varphi_{j}(x, s), \\
u_{j}(x, s)=-\int_{0}^{T} \int_{B_{y}^{N}} K^{*}(x, s ; y, t) \varphi_{j}(y, t) d y d t, \quad j=1,2, \cdots, \\
\left\|u_{j}\right\|_{L^{\infty}} \leqq\|f\|_{L^{\infty}} \quad j=1,2, \cdots, \\
\lim _{j \rightarrow \infty} u_{j}(x, s)=u(x, s) \quad(x, s) \in B^{N} \times[0, T],
\end{gathered}
$$

from where (4.5) follows. We get the continuity of $u(x, s)$ as follows. For $0 \leqq s_{0}<s \leqq T$ and $x_{0}, x \in B^{N}$,

$$
\begin{aligned}
u(x, s)-u\left(x_{0}, s_{0}\right)= & -\int_{s}^{T} \int_{B^{N}} K^{*}(x, s ; y, t) f(y, t) d y d t \\
& +\int_{s_{0}}^{T} \int_{B^{N}} K^{*}\left(x_{0}, s_{0} ; y, t\right) f(y, t) d y d t \\
= & \int_{s}^{T} \int_{B^{N}}\left[-K^{*}(x, s ; y, t)+K^{*}\left(x_{0}, s_{0} ; y, t\right)\right] f(y, t) d y d t \\
& +\int_{s_{0}}^{s} \int_{B^{N}} K^{*}\left(x_{0}, s_{0} ; y, t\right) f(y, t) d y d t=I_{1}+I_{2} .
\end{aligned}
$$

By (4.4) the last term $I_{2}$ will be arbitrarily small if we close $s$ and $s_{0}$ sufficiently. We have for small $\delta>0$ 


$$
\begin{aligned}
\left|I_{1}\right| \leqq & \int_{s}^{s+\delta} \int_{B^{N}}\left|K^{*}(x, s ; y, t)-K^{*}\left(x_{0}, s_{0} ; y, t\right)\right||f(y, t)| d y d t \\
& +\int_{s+\delta}^{T} \int_{B^{N}}\left|K^{*}(x, s ; y, t)-K^{*}\left(x_{0}, s_{0} ; y, t\right)\right||f(y, t)| d y d t
\end{aligned}
$$

The first term in the right hand side becomes arbitrarily small if we take $\delta$ (independent of $(x, s) \in B^{N} \times[0, T]$ ) sufficiently small. Finally for any fixed $\delta>0$, we have $K^{*}(x, s ; y, t)-K^{*}\left(x_{0}, s_{0} ; y, t\right) \rightarrow 0$ uniformly in $(x, s),(y, t) \in B^{N} \times[0, T]$ as $(x, s) \rightarrow\left(x_{0}, s_{0}\right)$ if $t-s_{0} \geqq \delta$ by virtue of (4.2).

Now we shall consider the general case where $u$ and $f$ are as in Theorem 4.1. For any $\left(x_{0}, s_{0}\right) \in B^{N} \times(0, T)$, take a function $\alpha(x, t) \in$ $C_{0}^{\infty}\left(B^{N} \times(0, T)\right)$ with $a \equiv 1$ in a neighbourhood of $\left(x_{0}, s_{0}\right)$. Then by the argument given above the function

$$
u_{\alpha}(x, s)=-\int_{0}^{T} \int_{B^{N}} K^{*}(x, s ; y, t) \alpha(y, t) f(y, t) d y d t
$$

is continuous in $B^{N} \times(0, T)$. On the other hand we have

$$
\left(L+\frac{\partial}{\partial s}\right)\left(u-u_{\alpha}\right)=f-\alpha f=(1-\alpha) f \quad \text { in } B^{N} \times(0, T) .
$$

Since $L+\frac{\partial}{\partial s}$ is hypoelliptic in $B^{N} \times(0, T)$, we have $u-u_{\alpha}$ is infininitely differentiable in a neighbourhood of $\left(x_{0}, t_{0}\right)$ where $\alpha \equiv 1$. We get Theorem 4.1 since $\left(x_{0}, t_{0}\right)$ is taken arbitrarily in $B^{N} \times(0, T)$.

\section{$\S 5$. The Cauchy problem}

Under the assumptions I, II and III, we first consider the Cauchy problem :

$$
\begin{gathered}
L u(x, s)+\frac{\partial}{\partial s} u(x, s)=0 \quad \text { in } \boldsymbol{R}^{n} \times(0, T), \\
\lim _{s>T} u(x, s)=\varphi(x) \quad \text { in } \mathscr{D}^{\prime}\left(\boldsymbol{R}^{n}\right),
\end{gathered}
$$

where $L$ is the operator given in $\S 1$.

THEOREM 5.1. Let $\varphi(x)$ be a bounded measurable function in $\boldsymbol{R}^{n}$, then the solution of the Cauchy problem (5.1), (5.2) is given by

$$
\begin{aligned}
u(x, s)=E_{x, s}[\varphi(\xi(T))]=\int_{\boldsymbol{R}^{n}} K^{*}(x, s ; y, t) \varphi(y) d y, & \\
& (x, s) \in \boldsymbol{R}^{n} \times(0, T) .
\end{aligned}
$$


We have $u \in C^{\infty}\left(\boldsymbol{R}^{n} \times(0, T)\right)$ and

$$
\sup _{\boldsymbol{R}^{n} \times(0, T)}|u(x, s)| \leqq \sup _{\boldsymbol{R}^{n}}|\varphi(x)|
$$

Furthermore if $\varphi(x)$ is continuous at $x_{0} \in \boldsymbol{R}^{n}$, then

$$
u(x, s) \rightarrow \varphi\left(x_{0}\right) \quad \text { as }(x, s) \rightarrow\left(x_{0}, T\right)
$$

Hence, if $\varphi(x)$ is a bounded continuous function, $u(x, s)$ is a classical solution of (5.1) and (5.2).

Remark 5.1. The uniqueness of the solution of the problem (5.1) and (5.2) follows from the Itô-formula and the assumptions I, II and III.

Proof of Theorem 5.1. The second equation in (5.3) is obtained by the standard argument by using (2.9). For any bounded measurable $\varphi(x)$ with compact support in $\boldsymbol{R}^{n}$,

$$
u(x, s)=\int_{R^{n}} K^{*}(x, s ; y, T) \varphi(y) d y
$$

is a solution of (5.1) and we have $u \in C^{\infty}\left(\boldsymbol{R}^{n} \times(0, T)\right)$ by (2.11) and the assumption III. Now let $\varphi(x)$ be merely bounded measurable function in $\boldsymbol{R}^{n}$. Let

$$
\varphi_{m}(x)=\left\{\begin{array}{cl}
\varphi(x) & |x| \leqq m, \\
0 & |x|>m, m=1,2, \cdots
\end{array}\right.
$$

and

$$
\begin{aligned}
u_{m}(x, s)=E_{x, s}\left[\varphi_{m}(\xi(T))\right]=\int_{\boldsymbol{R}^{n}} K^{*}(x, s ; y, T) \varphi_{m}(\xi) d \xi & \\
& (x, s) \in \boldsymbol{R}^{n} \times(0, T) .
\end{aligned}
$$

Then for each $(x, s) \in \boldsymbol{R}^{n} \times(0, T)$ we have

$$
u_{m}(x, s) \rightarrow u(x, s)=\int_{R^{n}} K^{*}(x, s ; y, T) \varphi(y) d y \quad \text { as } m \rightarrow \infty
$$

and we have

$$
\begin{aligned}
& \left(L+\frac{\partial}{\partial s}\right) u_{m}(x, s)=0 \quad \text { in } \boldsymbol{R}^{n} \times(0, T), \\
& \sup _{\boldsymbol{R}^{n} \times(0, T)}\left|u_{m}(x, s)\right| \leqq \sup _{\boldsymbol{R}^{n}}|\varphi(x)|, \quad m=1,2, \cdots:(2.10) \text {, }
\end{aligned}
$$


Hence by the Lebesgue bounded convergence theorem $u_{m} \rightarrow u$ in $L_{1 \mathrm{oc}}^{1}\left(\boldsymbol{R}^{n} \times[0, T]\right)$ as $m \rightarrow \infty$, which means $u_{m} \rightarrow u$ in $\mathscr{D}^{\prime}\left(\boldsymbol{R}^{n} \times[0, T]\right)$ as $m \rightarrow \infty$. By the assumption III and by (5.6), we have $u \in C^{\infty}\left(\boldsymbol{R}^{n} \times(0, T)\right)$ and $L u+\frac{\partial}{\partial s} u=0$ in $R^{n} \times(0, T)$.

The estimate (5.4) follows from (5.7).

By Theorem 2.1 we have

$$
K^{*}(x, s ; y, T) \rightarrow \delta(x-y) \quad \text { as } s \rightarrow T \quad \text { in } \mathscr{D}^{\prime}\left(R_{x}^{n}\right)
$$

for any $y \in \boldsymbol{R}^{n}$, whence we have $u(x, s) \rightarrow \varphi(x)$ in $\mathscr{D}^{\prime}\left(\boldsymbol{R}^{n}\right)$ as $s / T$.

Finally, let $x_{0} \in \boldsymbol{R}^{n}$ be a point of continuity for $\varphi(x)$. Let us remember that

$$
\xi_{x, s}(T)=x+\int_{s}^{T} b\left(\xi_{x, t}(\lambda), \lambda\right) d \lambda+\int_{s}^{T} \sigma\left(\xi_{x, s}(\lambda), \lambda\right) d w(\lambda)
$$

From (5.8) we have easily

$$
E\left|\xi_{x, s}(T)-x_{0}\right| \leqq C\left(\left|x-x_{0}\right|+(T-s)^{1 / 2}\right)
$$

for some positive constant $C$ independent of $x$ and $s$. Hence $\xi_{x, s}(T) \rightarrow$ $x_{0}$ in probability as $x \rightarrow x_{0}$ and $s \rightarrow T$, i.e. for any $\delta>0$,

$$
P\left(\left|\xi_{x, s}(T)-x_{0}\right|>\delta\right) \rightarrow 0 \quad \text { as } x \rightarrow x_{0}, t \rightarrow T .
$$

On the other hand for any $\varepsilon>0$, there exists $\delta>0$ such that

$$
\left|\varphi(x)-\varphi\left(x_{0}\right)\right| \leqq \varepsilon \quad \text { if }\left|x-x_{0}\right| \leqq \delta .
$$

Thus we have

$$
\begin{aligned}
\left|u(x, s)-\varphi\left(x_{0}\right)\right| & =\mid E_{x, s}\left[\varphi(\xi(T))-E_{x, s}\left[\varphi\left(x_{0}\right)\right] \mid\right. \\
& \leqq E\left|\varphi\left(\xi_{x, s}(T)\right)-\varphi\left(x_{0}\right)\right| \\
& \leqq \sup |\varphi| \cdot P\left(\left|\xi_{x, s}(T)-x_{0}\right| \geqq \delta\right)+\varepsilon .
\end{aligned}
$$

By (5.10) and since $\varepsilon$ is arbitrarily taken, the left hand side can be made arbitrarily small as $(x, s) \rightarrow\left(x_{0}, T\right)$. Consequently $u$ is continuous at $\left(x_{0}, 0\right)$. Q.E.D.

Next we shall consider the Cauchy problem:

$$
\begin{array}{cc}
L u+\frac{\partial u}{\partial s}=f(x, s) & \text { in } \boldsymbol{R}^{n} \times(0, T), \\
u(x, 0)=0 & \text { on } \boldsymbol{R}^{n} .
\end{array}
$$


THEOREM 5.2. Let $f(x, t)$ be a bounded measurable function in $\boldsymbol{R}^{n} \times(0, T)$, then the solution of the Cauchy problem (5.11), (5.12) is given by

$$
\begin{aligned}
u(x, s) & =-E_{x, s} \int_{s}^{T} f(\xi(t), t) d t \\
& =-\int_{s}^{T} \int_{R^{n}} K^{*}(x, s ; y, t) f(y, t) d y d t .
\end{aligned}
$$

We have $u \in C\left(\boldsymbol{R}^{n} \times[0, T]\right)$ and

$$
|u(x, s)| \leqq(T-s) \sup _{\boldsymbol{R}^{n} \times(0, T)}|f(x, t)|, \quad(x, s) \in \boldsymbol{R}^{n} \times(0, T) .
$$

Therefore $u(x, s)$ satisfies (5.12) in the ordinary sense.

Proof. The second equation in (5.13) is obvious by (5.3). The estimate (5.14) follows from (5.13) and (2.10). Now let $f(x, t)$ be a bounded measurable function in $\boldsymbol{R}^{n} \times[0, T]$ and let

$$
f_{m}(x, t)=\left\{\begin{array}{cl}
f(x, t) & |x| \leqq m, \\
0 & |x|>m, m=1,2, \cdots .
\end{array}\right.
$$

Then, as in the proof of Theorem 4.1, the functions

$$
u_{m}(x, s)=-\int_{s}^{T} \int_{R^{n}} K^{*}(x, s ; y, t) f_{m}(y, t) d y d t, \quad m=1,2, \cdots
$$

satisfy (5.11) with $f=f_{m}$ in the weak sense and (5.12) in the ordinary sense. We have $u_{m}(x, s) \rightarrow u(x, s)$ as $m \rightarrow \infty$ and

$$
\left|u_{m}(x, s)\right| \leqq T \sup _{\boldsymbol{R}^{n} \times[0, T]}|f(x, s)|, \quad m=1,2, \cdots
$$

By the Lebesgue convergence theorem we have $u_{m}(x, s) \rightarrow u(x, s)$ in $\mathscr{D}^{\prime}\left(\boldsymbol{R}^{n} \times[0, T]\right)$ as $m \rightarrow \infty$, hence $u$ satisfies (5.11) in the weak sense and $u$ is continuous in $R^{n} \times[0, T]$ by Theorem 4.1 .

Now let $c(x, s)$ be a bounded measurable function in $\boldsymbol{R}^{n} \times[0, T]$. We shall finally consider the Cauchy problem:

$$
L u+c(x, s) u+\frac{\partial u}{\partial s}=f(x, s) \quad \text { in } \boldsymbol{R}^{n} \times(0, T),
$$

$$
\lim _{s / T} u(x, s)=\varphi(x) \quad \text { in } \mathscr{D}^{\prime}\left(\boldsymbol{R}^{n}\right)
$$


THEOREM 5.3. Let $f(x, s)$ and $\varphi(x)$ be bounded measurable functions defined in $\boldsymbol{R}^{n} \times[0, T]$ and in $\boldsymbol{R}^{n}$ respectively. Then the solution of (5.15) and (5.16) is given by

$$
\begin{aligned}
u(x, s)= & E_{x, s} \varphi(\xi(T)) \exp \left[\int_{s}^{T} c(\xi(\lambda), \lambda) d \lambda\right] \\
& -E_{x, s} \int_{s}^{T} f(\xi(t), t) \exp \left[\int_{s}^{t} c(\xi(\lambda), \lambda) d \lambda\right] d t \\
= & \int_{R^{n}} \Gamma^{*}(x, s ; y, t) \varphi(y) d y \\
& -\int_{s}^{T} \int_{R^{n}} \Gamma^{*}(x, s ; y, t) f(y, t) d y d t, \quad(x, s) \mathbf{R}^{n} \times(0, T),
\end{aligned}
$$

where $\Gamma^{*}$ is the fundamental solution for $L+c+\frac{\partial}{\partial s}$ constructed in §3. $u(x, s)$ is continuous in $\boldsymbol{R}^{n} \times[0, T]$ and we have by (3.9)

$$
|u(x, s)| \leqq e^{(T-s) M}\left(\sup _{\boldsymbol{R}^{n}}|\varphi(x)|+(T-s) \sup _{\boldsymbol{R}^{n} \times[0, T]}|f(x, s)|\right),
$$

where $M=\sup _{\boldsymbol{R}^{n} \times[0, T]} c(x, s)$.

Furthermore if $\varphi(x)$ is continuous at $x_{0} \in \boldsymbol{R}^{n}$, then we have

$$
u(x, s) \rightarrow \varphi\left(x_{0}\right) \quad \text { as }(x, s) \nearrow\left(x_{0}, T\right) .
$$

Proof. We set

$$
u(x, s)=\int_{R^{n}} \Gamma^{*}(x, s ; y, t) \varphi(y) d y-\int_{s}^{T} \int_{R^{n}} \Gamma^{*}(x, s ; y, t) f(y, t) d y d t .
$$

Then by almost the same way as in the proof of Theorem 5.1 and 5.2 and by using the estimate (3.9) we can show that $u(x, s)$ is a solution of (5.15) and (5.16). $u(x, s)$ is continuous in $\boldsymbol{R}^{n} \times[0, T]$ by Theorem 4.1. It remains to prove the equality of two expressions in (5.17). We shall merely prove the case where $f \equiv 0$, i.e.

$u(x, s)=E_{x, s} \varphi(\xi(T)) \exp \left[\int_{s}^{T} c(\xi(\lambda), \lambda) d \lambda\right]=\int_{R^{n}} \Gamma^{*}(x, s ; y, t) \varphi(y) d y$.

The remaining part can be proved similarly. First take two series of functions such that

$$
\begin{gathered}
c_{m}(x, s) \in C^{2}\left(\boldsymbol{R}^{n} \times[0, T]\right), \quad \varphi_{m}(x) \in C^{2}\left(\boldsymbol{R}^{n}\right), \\
\left\|c_{m}(x, s)\right\|_{L^{\infty}} \leqq\|c(x, s)\|_{L^{\infty}}, \quad\left\|\varphi_{m}(x)\right\|_{L^{\infty}} \leqq\|\varphi(x)\|_{L^{\infty}} \quad m=1,2, \cdots,
\end{gathered}
$$




$$
\begin{gathered}
\lim _{m \rightarrow \infty} c_{m}(x, s)=c(x, s) \quad(x, s) \in \boldsymbol{R}^{n} \times[0, T], \\
\lim _{m \rightarrow \infty} \varphi_{m}(x)=\varphi(x) \quad x \in \boldsymbol{R}^{n} .
\end{gathered}
$$

By the results of [8], there exists $C^{2}$-solution $u_{m}$ of the problem

$$
L u+c_{m}(x, s) u+\frac{\partial u}{\partial s}=0 \quad \text { in } R^{n} \times(0, T),
$$

$$
u(x, T)=\varphi_{m}(x) \quad \text { in } R^{n} .
$$

As an application of Itô-formula to the function

$$
u_{m}\left(\xi_{x, s}(\lambda), \lambda\right) \exp \left[\int_{s}^{\lambda} c(\xi(\sigma), \sigma) d \sigma\right]
$$

and the process (2.5), we have

$$
u_{m}(x, s)=E_{x, s} \varphi_{m}(\xi(T)) \exp \left[\int_{s}^{T} c_{m}(\xi(\lambda), \lambda) d \lambda\right]
$$

We can easily show by using the Lebesgue convergence theorem that for any Brownian motion $w$

$$
\varphi_{m}\left(\xi_{x, s}(T)\right) \exp \left[\int_{s}^{T} c_{m}\left(\xi_{x, s}(\lambda), \lambda\right) d \lambda\right]
$$

converges boundedly (in $w$ ) to

$$
\varphi\left(\xi_{x, s}(T)\right) \exp \left[\int_{s}^{T} c\left(\xi_{x, s}(\lambda), \lambda\right) d \lambda\right] .
$$

Hence we have for each $(x, s) \in \boldsymbol{R}^{n} \times[0, T]$,

$$
u_{m}(x, s) \rightarrow u(x, s)=E_{x, s} \varphi(\xi(T)) \exp \left[\int_{s}^{T} c(\xi(\lambda), \lambda) d \lambda\right] \quad m \rightarrow \infty .
$$

Obviously this is a bounded convergence in $(x, s) \in R^{n} \times(0, T)$. Hence $u$ satisfies (5.15) with $f \equiv 0$ in the sense of distributions. (5.16) is proved as in the proof of Theorem 5.1.

\section{§6. Remark on the Fichera problem}

Let

$$
L=\sum_{i, j=1}^{n} a_{i j}(x) \frac{\partial^{2}}{\partial x_{i} \partial x_{j}}+\sum_{j=1}^{n} b_{j}(x) \quad x \in \boldsymbol{R}^{n}
$$


be independent of $t$ and satisfy the assumptions in Theorem 2.2. Let $G$ be a bounded domain in $\boldsymbol{R}^{n}$ and for simplicity suppose the boundary $\partial G$ is smooth: $\partial G \in C^{3}$. As in [9], we denote by $\Sigma_{2}$ the set of $\partial G$ where

$$
b(x) \equiv \sum_{j, k=1}^{n}\left(b_{j}(x)-\frac{\partial a_{i j}}{\partial x_{k}}\right) \nu_{j}<0,
$$

where $\left(\nu_{1}, \cdots, \nu_{n}\right)$ is the interior normal vector at $x \in \partial G$. The set of boundary points where

$$
\sum_{i, j=1}^{n} a_{i j}(x) \xi_{i} \xi_{j}>0 \quad \xi \in R^{n}, \xi \neq 0
$$

will be denoted by $\Sigma_{3}$. Let $c(x)$ be a bounded measurable function in $G$ and $c(x) \leqq 0 x \in G$. We shall consider the first boundary value problem :

$$
\begin{gathered}
L u+c u=f \quad \text { in } G, \\
u=g \quad \text { on } \Sigma_{23} \equiv \Sigma_{2} \cup \Sigma_{3},
\end{gathered}
$$

where $f$ is a given bounded measurable function in $G$ and $g$ is a given continuous function on $\Sigma_{23}=\Sigma_{2} \cup \Sigma_{3}$.

Under the assumption that either $c(x)$ be uniformly negative or

$$
\sup _{x \in G} E_{x} \tau<\infty,
$$

where $\tau=\inf \left\{t \geqq 0 ; \xi_{x, 0}(t) \notin G\right\}$, the solution satisfying (6.1) in the sense of distributions and (6.2) in the ordinary sense is given by

$$
\begin{aligned}
u(x)= & E_{x} g(\xi(\tau)) \exp \left[\int_{0}^{\tau} c(\xi(s)) d s\right] \\
& -E_{x} \int_{0}^{\tau} f(\xi(\tau)) \exp \left[\int_{0}^{t} c(\xi(s)) d s\right] d t \quad \text { (c.f. [11]). }
\end{aligned}
$$

By applying our results obtained in $\S 4$, we have $u(x) \in C\left(G \cup \Sigma_{23}\right)$. Furthermore by (6.3) and (6.4) we have the estimate

$$
\|u\|_{\infty, G} \leqq C\left(\|g\|_{\infty, \Sigma_{23}}+\|f\|_{\infty, G}\right),
$$

where $C$ is a constant independent of $f$ and $g$.

\section{REFERENCES}

[1] Bony, J.-M., Principe du maximum. Inegalité de Harnack et unicité du problème de Cauchy pour les opérateurs elliptiques dégénérés, Ann. Inst. Fourier, Grenoble 19(1) (1969), 277-304. 
[2] Freidlin, M. I., On the factorization of nonnegative definite matrices, Theor. Probability Appl. 13 (1968), 354-356.

[3] Friedman, A., Partial differential equations of parabolic type, Prentice-Hall (1964).

[4] - Stochastic differential equations and applications, Vol.1,2, Academic Press (1975).

[ 5] - Fundamental solutions for degenerate parabolic equations, Acta Math. 133 (1974), 171-217.

[6 ] Ichihara, K. and Kunita, H., A classification of the second order degenerate elliptic operators and its probabilistic characterization, Z. Wahrscheinlichkeitstheorie verw. Gebiete 30 (1974), 235-254.

[ 7 ] Matsuzawa, T., On some degenerate parabolic equations I, Nagoya Math. J. 51 (1973), 57-77, II, Nagoya Math. J. 52 (1973), 61-84.

[ 8 ] Oleinik, O. A., Alcuni risultati sulle equazioni lineari elliptico-paraboliche a derivate parziali del secondo ordine, Rend. Classe, Sci. Fis. Mat. Nat. Acad. Naz. Lincei, Ser. 8, Vol., 40 (1966), 775-784.

[9] Oleinik, O. A. and Radkevich, E. V., Second order equations with nonnegative characteristic form, American Math. Soc., Providence, 1973.

[10] Schwartz, L., Théorie des noyaux, Proc. International Congress of Mathematicians, Vol. 1, 220-230, American Math. Soc. Providence, 1952.

[11] Strook, D. and Varadhan, S. R. S., On degenerate elliptic-parabolic operators of second order and their associated diffusions, Comm. Pure Applied Math., Vol. 25 (1972), 651-713.

Department of Mathematics

Nagoya University 